\title{
ORIGINAL ARTICLE Alloreactivity: the Janus-face of hematopoietic stem cell transplantation
}

\author{
A Gratwohl ${ }^{1}$, A Sureda ${ }^{2}$, J Cornelissen ${ }^{3}$, J Apperley ${ }^{4}$, P Dreger ${ }^{5}$, R Duarte $^{6}$, HT Greinix $^{7}$, E Mc Grath ${ }^{8}$, N Kroeger ${ }^{9}$, F Lanza ${ }^{10}$, A Nagler ${ }^{11}$, \\ JA Snowden ${ }^{12}$, D Niederwieser ${ }^{13}$ and R Brand ${ }^{14}$ for the European Society for Blood and Marrow Transplantation (EBMT)
}

Differences in major and minor histocompatibility antigens between donor and recipient trigger powerful graft-versus-host reactions after allogeneic hematopoietic stem cell transplantation (HSCT). The clinical effects of alloreactivity present a Janus-face: detrimental graft-versus-host disease increases non-relapse mortality, beneficial graft-versus-malignancy may cure the recipient. The ultimate consequences on long-term outcome remain a matter of debate. We hypothesized that increasing donor-recipient antigen matching would decrease the negative effects, while preserving antitumor alloreactivity. We analyzed retrospectively a predefined cohort of 32838 such patients and compared it to 59692 patients with autologous HSCT as reference group. We found a significant and systematic decrease in non-relapse mortality with decreasing phenotypic and genotypic antigen disparity, paralleled by a stepwise increase in overall and relapse-free survival (Spearman correlation coefficients of cumulative excess event rates at 5 years $0.964 ; P<0.00$; respectively $0.976 ; P<0.00$ ). We observed this systematic stepwise effect in all main disease and disease-stage categories. The results suggest that detrimental effects of alloreactivity are additive with each step of mismatching; the beneficial effects remain preserved. Hence, if there is a choice, the best match should be donor of choice. The data support an intensified search for predictive genomic and environmental factors of 'no-graft-versus-host disease'.

Leukemia (2017) 31, 1752-1759; doi:10.1038/leu.2017.79

\section{INTRODUCTION}

For many patients with congenital or acquired severe hematological disorders, allogeneic hematopoietic stem cell transplantation (HSCT) offers unique potential for cure. Bone marrow and peripheral blood from family members or unrelated volunteer donors serve as a source of stem cells for syngeneic or allogeneic transplants. Worldwide annual numbers of allogeneic and autologous HSCT currently exceed sixty thousand and figures are still rising. ${ }^{1-4}$ Several elements contribute to the success of HSCT. The conditioning therapy preceding the transplant prepares the recipient (host) to accept the graft, and reduces tumor load. The infusion of healthy allogeneic hematopoietic stem cells restores hematopoiesis and installs an alloreactive immune surveillance of the tumor. Indeed, allogeneic HSCT provided the first proof of principle that immune mechanisms can eradicate malignant disease. However, a double immunological barrier adds complexity, including host-versus-graft (graft rejection) and graftversus-host reactions. ${ }^{5,6}$ Both barriers increase with the degree of disparities in major and minor histocompatibility antigens. Graft rejection is always associated with detrimental effects for the patient. $^{5-9}$ In contrast, the graft-versus-host reactions present a Janus face. The detrimental part, graft-versus-host disease, remains the most important direct or indirect cause for nonrelapse mortality after allogeneic $\mathrm{HSCT}^{1,5-7}$ The 'other face', the graft-versus-malignancy effect, is desired and may result in cure of the recipient. ${ }^{10}$

HSCT presents also as role model of precision medicine: one individual donor is selected for one individual recipient based on genetic findings. Best donor choice and best matching for minor and major histocompatibility antigens to modulate the two divergent alloreactive effects have been a matter of debate since the very beginning. ${ }^{11}$ This discussion has intensified in recent years. The lack of a Human Leukocyte Antigen (HLA)-identical sibling donor for many patients has triggered the search for alternative donors, including mismatched family members or partially matched unrelated donors. ${ }^{12-15}$ The degree of matching needed for an 'acceptable' unrelated donor is still not settled. Some approaches postulate' a donor option' for every patient. ${ }^{16}$ Indeed, the ready availability and the promising early results have led to a substantial increase in the use of haploidentical mismatched family-donor transplants. ${ }^{12,17}$ Results from large series and with a long follow-up, however, are lacking. In this context, and in view of the need for better data in the present precision medicine initiative, ${ }^{18}$ we analyzed a predefined large cohort of patients with a confirmed follow-up of 8 years. ${ }^{19}$ We hypothesized a donor type hierarchy based on phenotypic and genotypic differences between donor and recipient in major (defined by HLA) and minor (defined by HY) histocompatibility

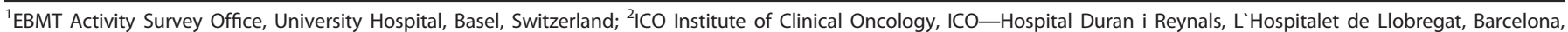

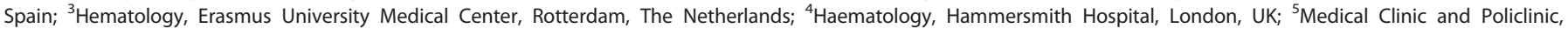

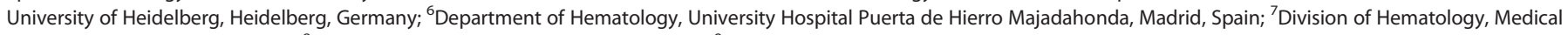

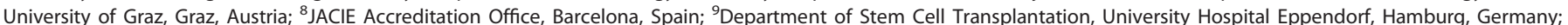

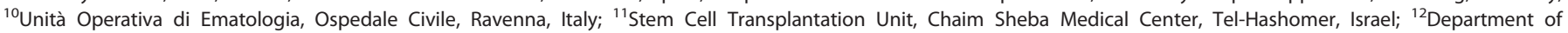

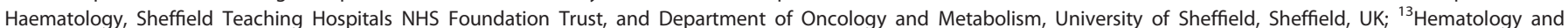

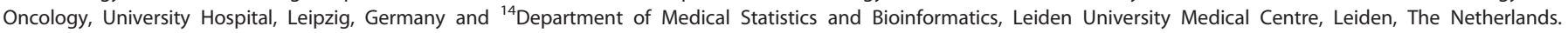
Correspondence: Professor A Gratwohl, University Hospital Basel, Petersgraben 4, EBMT activity survey office, Basel CH-4031, Switzerland. 
antigens and analyzed non-relapse mortality, relapse-free and overall survival in specified donor type groups.

\section{MATERIALS AND METHODS}

\section{Study design and patient population}

This retrospective observational analysis is based on a previously published cohort of 102549 patients treated between 1999 and 2006 with HSCT for an acquired hematological malignancy from 1999 to 2006 (Table 1) and reported by 404 teams to the European Society for Blood and Marrow Transplantation (EBMT) database (www.ebmt.org). ${ }^{19}$ This corresponds to $93 \%$ of all patients transplanted during this period by the participating teams with these indications. The patient' survival data were updated as of 1 January 2015. The final analysis was restricted to patients with full information on all variables integrated into the analysis. It includes 32838 patients with a first allogeneic HSCT (35\%) and 59692 patients with autologous HSCT (65\%) as reference group (Total $N=92530$ patients). The main endpoint in all analyses was 'death' due to non-relapse mortality, leading to estimates for non-relapse mortality, overall survival and relapsefree survival in a competing risk framework. All the data were censored at 8 years post HSCT to provide for a homogeneous observation period.

All EBMT teams are required to obtain patients' consent and to have internal review board approval for their transplant programs and for data transfer to EBMT. The analysis did not use individual patient data; no ethics approval was mandated.

\section{Definitions}

Definitions relied on the pre-specified hypothesis that increasing phenotypic and genotypic matching for minor and major histocompatibility antigens would be associated with decreasing effects of graft-versushost but preserve antitumor activity. Donor type was used as predefined in the EBMT database and as reported by the transplant teams, split into syngeneic donor, HLA-identical sibling donor, matched other family donor, matched unrelated donor, mismatched unrelated donor and mismatched family donor (Table 2). Specific HLA-antigens were not collected in this database, but 'donor type' served as a surrogate marker for the numbers of potential phenotypic and genotypic mismatches for major and minor histocompatibility antigens. For the latter, we used the donor-recipient gender combination and defined the female donor-male recipient combination as $\mathrm{HY}^{+}$and all other donor-recipient gender combinations as $\mathrm{HY}^{-}$. By definition, $\mathrm{HY}$ status is $\mathrm{HY}^{-}$for autologous and syngeneic transplants. HY status had no significant additional effect in mismatched related on unrelated transplants (data not shown); hence, these two donor types were not split by HY. Autologous HSCT served as reference group, since no alloreactivity occurs after such transplants.

\section{Statistical analysis}

The above-mentioned a priori ordering was provided to the statistician before the data were analyzed and was not modified in any respect during the analyses. The focus of the statistical approach was not to prove the significance of any specific risk (sub) factor in its association with the hazard of the outcomes but on the establishment of a pre-specified 'dose response relationship' in the framework of a model which would not impose such 'proportionality'. Hence, the use of a COX model with the a priori ordered risk categories is impossible. To maximize power of the analyses, a stratified COX model was used where each of the ordered risk categories has its own base line curve. Since then by definition the Hazard Ratio comparing the 10 a priori specified categories would not be retained by the model to be constant, comparison of those categories is impossible using a single Hazard Ratio. We integrated previously described standard risk factors ${ }^{20,21}$ and used the cumulative hazard estimates in the stratified models. ${ }^{22}$ The model characterizes the differences between the 10 categories and allows their ordering to arbitrarily change over follow-up time. Survival curves over an 8-year period were modeled using donor type as a predefined stratification variable.

All models contained previously established key risk factors as continuous or factorized covariates ${ }^{20,21}$ (Supplementary Figure 1): main disease (acute leukemia, chronic leukemia, myelodysplastic syndrome/ myeloproliferative neoplasias, lymphoma and plasma cell disorders; Supplementary Figure 1a), disease stage (good risk, intermediate, poor risk; Supplementary Figure 1b), recipient age (Supplementary Figure 1c) donor age (allogeneic HSCT only, Supplementary Figure 1d) and. conditioning (reduced intensity conditioning/standard conditioning;
Supplementary Figure 1e) and, T-cell depletion (TCD- vs TCD+). As microeconomic variables (covariates at the center level), we used center patient volume and center program duration. We used both as continuous variables in the model but recoded them into 5 categories to avoid problems with outliers. ${ }^{19}$ Calendar year was used as a continuous covariate. Relapse, as provided by the database and non-relapse mortality were acknowledged as competing risks.

We used the cumulative hazard or-more generically speaking-the cumulative event rate as the primary measure to compare the various ordered risk categories. The difference in the cumulative event rates was denoted by the word 'cumulative excess event rate' (CEER), in strict analogy to the notion of 'force of mortality'22 in the case of the outcome being death. Therefore, our CEER can be interpreted as 'excess mortality rate'. The CEER does not measure a cumulative incidence of the outcome itself.

For statistical testing of the null hypothesis of 'no ordering over the 10 donor types' versus the alternative hypothesis 'systematic increase/ decrease of a cumulative event rate', we used a non-parametric Spearman correlation coefficient between the CEER and the order number of the risk category, excluding the reference category. All analyses were performed by SPSS version 22/24.

\section{RESULTS AND DISCUSSION}

Patient population

The analysis comprised 32838 recipients of allogeneic HSCT and 59692 patients with autologous HSCT as reference group (Table 1). In this heterogeneous population, allogeneic HSCT was preferentially used for acute leukemias ( $N=19554 ; 76 \%$ allogeneic), chronic leukemias ( $N=6698 ; 83 \%$ allogeneic) and myelodysplastic/myeloproliferative disorders ( $N=3122 ; 94 \%$ allogeneic); autologous HSCT was preferentially used for lymphoma ( $N=29476 ; 91 \%$ autologous) and plasma cell disorders ( $N=22582 ; 97 \%$ autologous).

The 10 groups of donor types, defined by their degree of phenotypic and genotypic matching for minor and major histocompatibility antigens (see Table 2 for details) differed significantly in size. They included syngeneic $(N=201 ; 0.2 \%)$; $\mathrm{HLA}$-identical, $\mathrm{HY}^{-}$sibling (for definitions of $\mathrm{HY}^{-/+}$see methods) $(N=13644 ; 15 \%) ; \quad H L A$-identical, $\mathrm{HY}^{+}$sibling $(N=4593 ; 5 \%)$; matched, $\mathrm{HY}^{-}$family $(\mathrm{N}=542 ; 0.6 \%)$; matched, $\mathrm{HY}^{+}$family $(N=183 ; 0.2 \%)$; matched, $\mathrm{HY}^{-}$unrelated $(N=7667 ; 8 \%)$; matched, $\mathrm{HY}^{+}$unrelated $(N=1322 ; 1 \%)$; mismatched unrelated $(N=3259$; $4 \%)$, and mismatched related donors ( $N=1427 ; 2 \%$; Table 2$)$. The donor groups differed significantly with respect to disease, disease stage, conditioning and T-cell depletion status (Table 1). There were significant differences between the 404 participating HSCT centres from 25 European countries regarding their center experience, patient volume and accreditation status. There were significant changes in the indications for HSCT over time. ${ }^{19}$

\section{'Standard' risk factors and outcome}

The analysis confirmed previously defined risk factors. ${ }^{20,21}$ Outcome differed between the five main disease categories. Survival estimates for patients with autologous and allogeneic HSCT showed non-proportional hazards for the main disease categories (Supplementary Figure 1a). Overall survival decreased from good risk to intermediate and poor risk disease-stage due to increasing relapse incidence and increasing non-relapse mortality in all disease categories and for all donor types (Supplementary Figure 1b).

Overall survival decreased with increasing recipient and donor age due to increasing non-relapse mortality after both, allogeneic and autologous HSCT (Supplementary Figures 1c and d). Transplant techniques such as reduced intensity conditioning were associated with overall survival in patients with allogeneic HSCT (Supplementary Figure 1e). 
Table 1. Demographics of 92530 patients treated by 407 European teams in 25 countries between 1999 and 2006 with an allogeneic $(N=32838$; $35 \%)$ or autologous ( $N=59692 ; 65 \%)$ HSCT

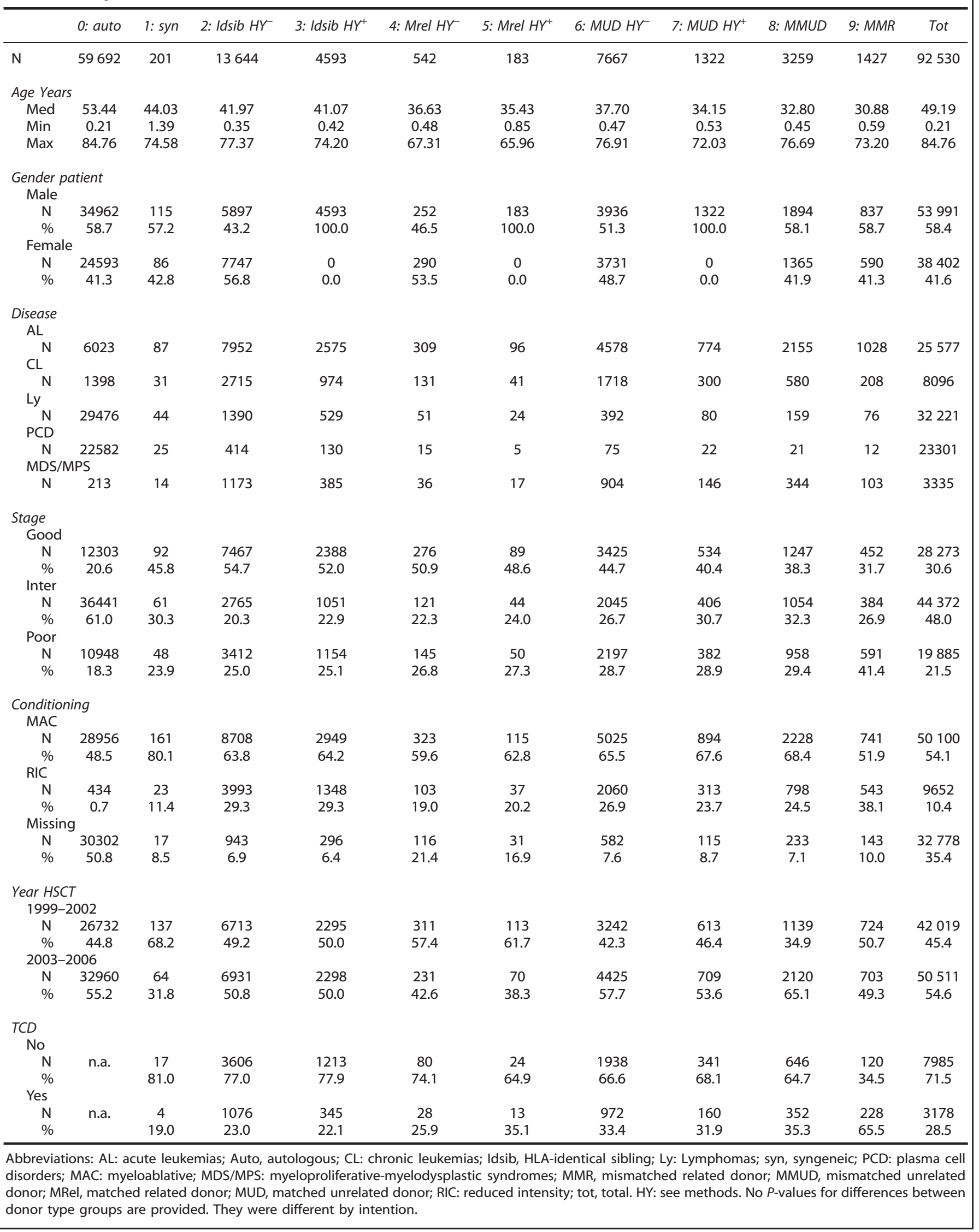


Table 2. Donor type definitions and degree of mismatches

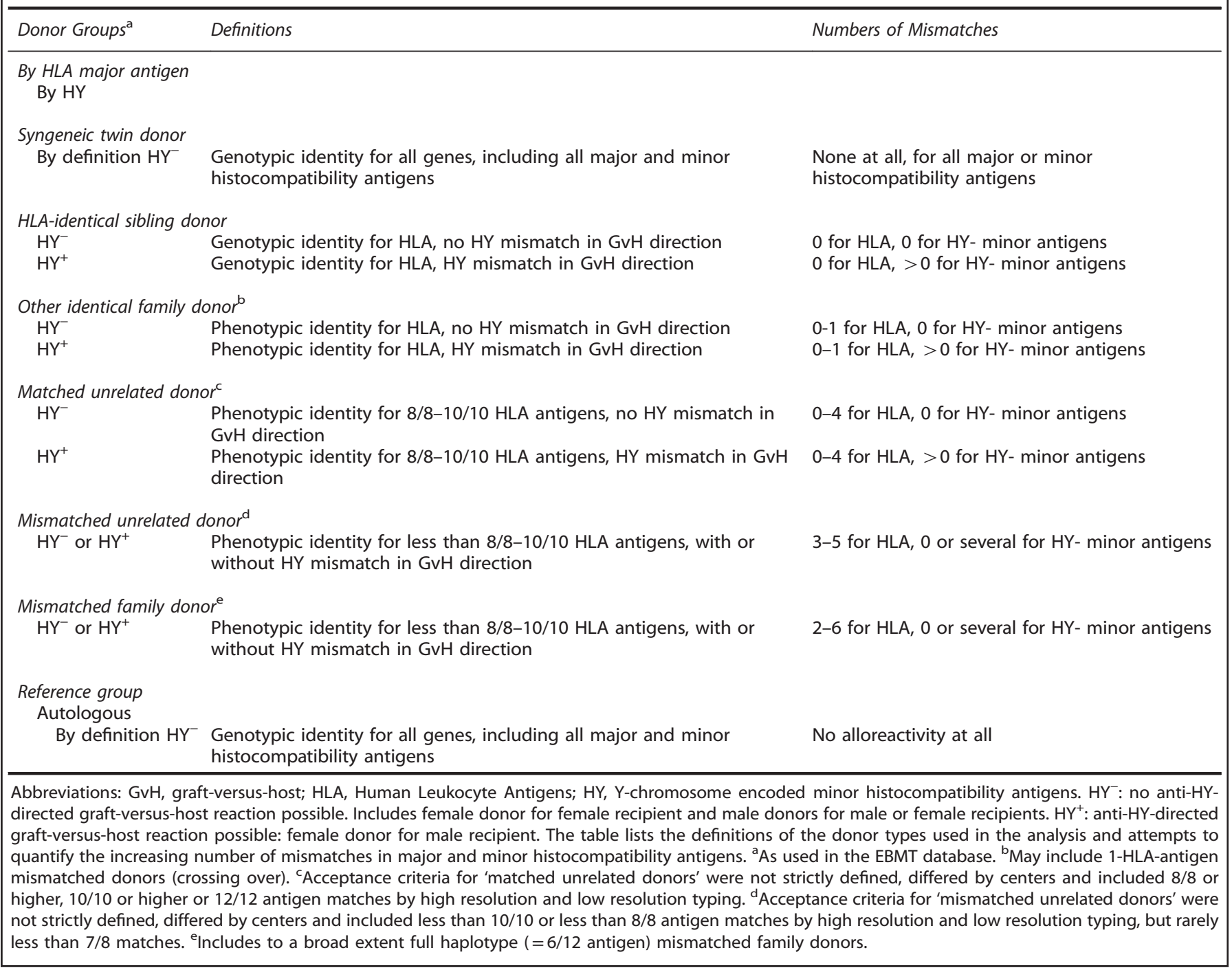

Degree of donor-recipient matching and outcome: donor type hierarchy

The data confirmed the hypothesis; they showed a clear donor type hierarchy. Non-relapse mortality increased significantly and systematically with each step of mismatching between donor and recipient, as shown by the cumulative event excess rate compared to the reference group autologous HSCT (CEER; Spearman coefficient 0.964; $P<0.001$; Figure 1a; Table 3$).^{22}$ In parallel, relapse-free and overall survival decreased by each step. Consequently, the cumulative excess event rate for death was lowest after syngeneic HSCT (CEER $=-0.10)$ and increased systematically and significantly from $\mathrm{HLA}$-identical, $\mathrm{HY}^{-}$siblings (CEER 0.04) to mismatched related (CEER 0.57) donor HSCT (Spearman coefficient for overall survival $0.976 ; P<0.001$; Table 3 ; Figure $1 \mathrm{~b}$ ). Probability of overall survival improved significantly and systematically with each step of matching from $31 \%$ at 5 years for the patients with a mismatched related donor HSCT to 54\% with an HLA-identical, $\mathrm{HY}^{-}$sibling donor transplant, and to $69 \%$ with a syngeneic HSCT (Figure 2; Table 3). Relapse-free survival increased as stepwise from $26 \%$ at 5 years with a mismatched related donor HSCT to $43 \%$ with an $\mathrm{HLA}$-identical $\mathrm{HY}^{-}$sibling donor transplant, and $55 \%$ for the 201 patients with a syngeneic HSCT (Table 3).
In summary, the data showed a systematic, monotonous decrease in the cumulative excess death rate and an increase in overall and relapse-free survival with each step of matching for $\mathrm{HLA}$, and in each $\mathrm{HY}^{-}$versus $\mathrm{HY}^{+}$constellation (Table 1; Figure 1). The donor type groups with no mismatch had the best overall long-term survival (Figure 2). Detailed subgroup analyses showed the same systematic effects of matching on overall survival and relapse-free survival for all main disease categories and the three disease stages (Supplementary Figure 2; Supplementary Tables $1 \mathrm{a}$ and $b$ ). The analyses confirmed the same systematic and stepwise effects of donor type on the cumulative excess event rates in overall survival, non-relapse mortality and relapse-free survival for all main disease categories and all three disease stages (Supplementary Tables 2a and b). Results showed the same effects when one disease category with large numbers of both, allogeneic and autologous HSCT was analyzed, acute myeloid leukemia (Spearman rank for overall survival 0.903; $P<0.000$ ). The Spearman rank correlation of the cumulative excess event rates for non-relapse mortality, overall and relapse-free survival confirmed the consistent and systematic association with donor type in each disease category (Supplementary Table 3a) and each disease stage (Supplementary Table 3b). 


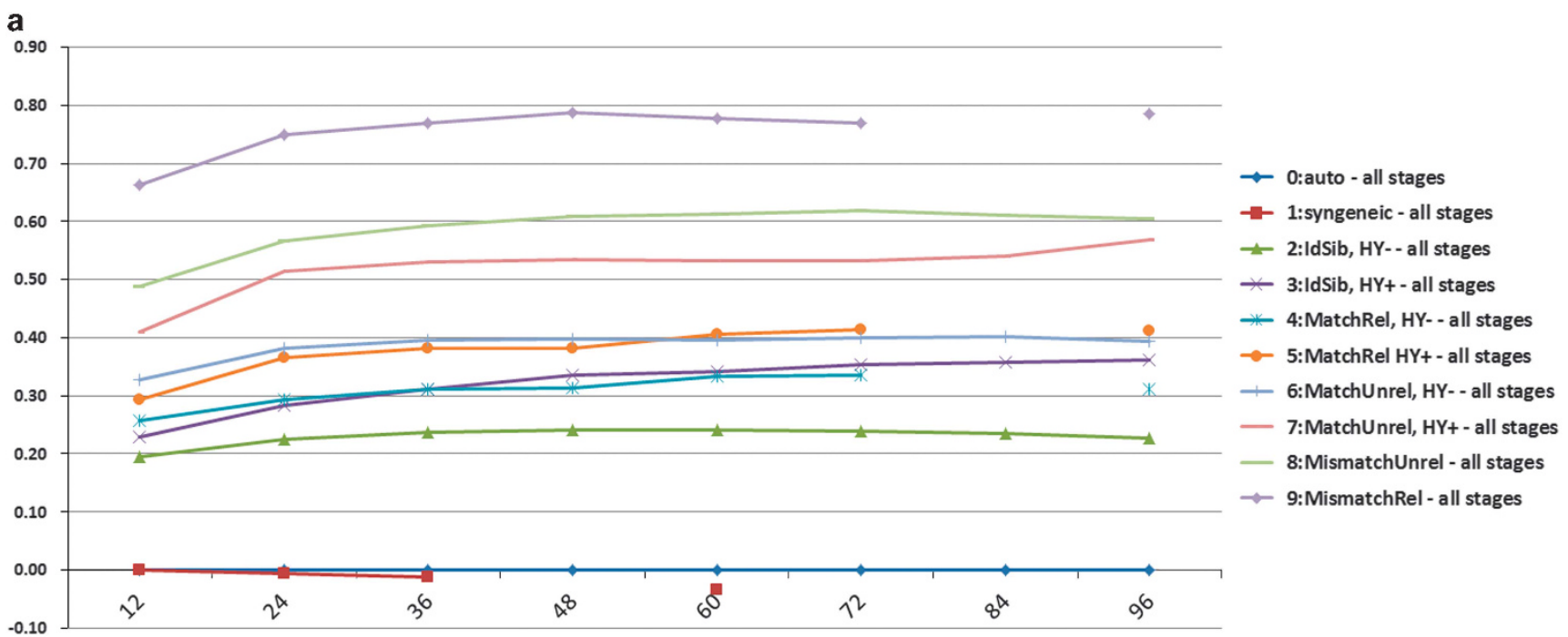

b

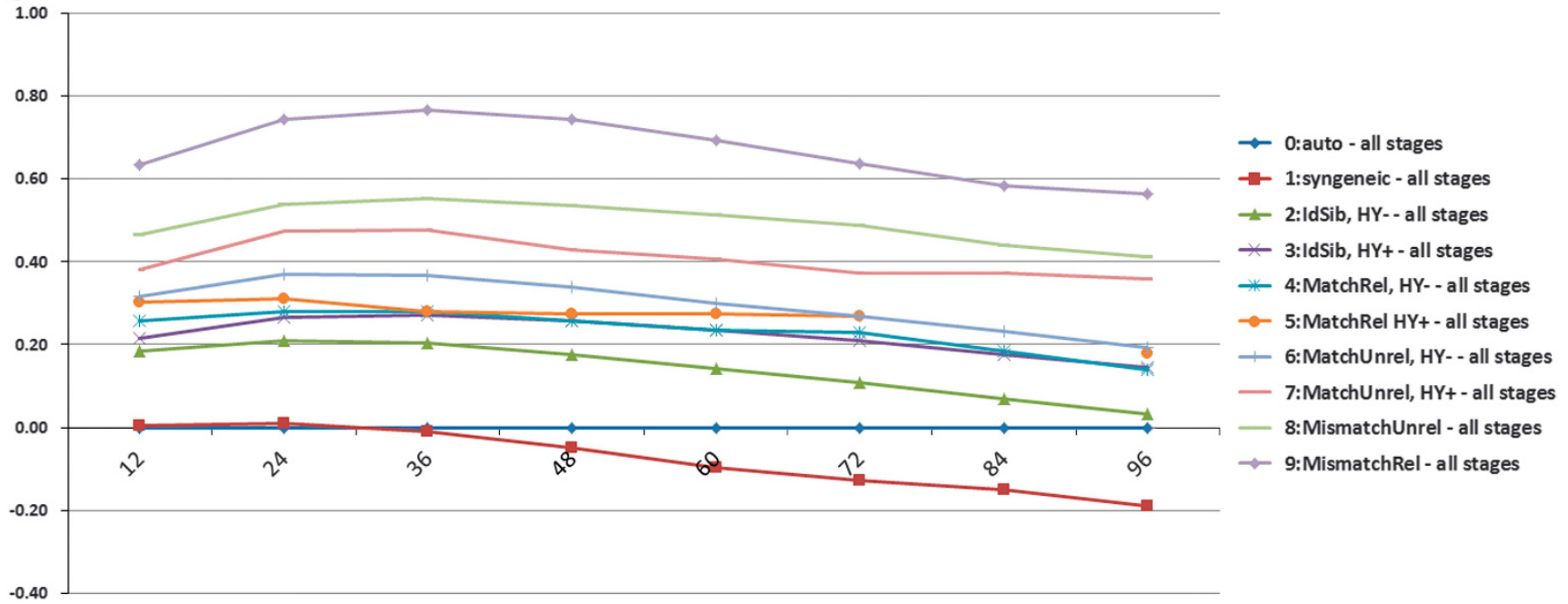

Figure 1. Donor type and cumulative excess event rates. The figures depict cumulative excess event rates by donor type (for definitions see methods section) of 32838 patients treated between 1999 and 2006 with an allogeneic HSCT in Europe relative to 59692 patients with an autologous HSCT. All estimates adjusted for calendar year, conditioning, disease, stage, center experience, center size and evaluated at covariate means. (a) Cumulative excess event rate of non-relapse death. The graph illustrates the stepwise increase in cumulative excess event rates of death due to non-relapse mortality with increasing mismatches in minor and major histocompatiblity antigens between donor and recipient (for definitions of donor type see methods section). (b) Cumulative excess event rate of death from any cause. The graph illustrates the stepwise increase in cumulative excess event rates of death from any cause with increasing mismatches in minor and major histocompatiblity antigens between donor and recipient (for definitions of donor type see methods section). There appears no compensation of mortality through reduced relapse rate.

Hence, the data showed a significant, systematic and stepwise decrease in non-relapse mortality with increasing phenotypic and genotypic matching between donor and recipient for minor and major histocompatibility antigens, accompanied by a parallel systematic improvement in relapse-free and overall survival. The findings confirmed our hypothesis. They suggest a basic principle of the Janus face alloreactivity in HSCT. The detrimental effects of graft-versus-host disease, which ultimately result in non-relapse mortality, increase in a cumulative pattern with each step of mismatching; the beneficial effects of relapse reduction remain maintained with matching. This fits with previous findings; negative patient, donor and disease-related pre-transplant risk factors act additively. ${ }^{20,21}$

There are some caveats and limitations. We had no detailed information on the exact numbers of disparities for HLA and minor antigens. We selected 'donor type' as reported and as defined by the EBMT data collection system. We used it as a marker for phenotypic and genotypic differences in HLA antigens between donor and recipient (www.ebmt.org). We used the female donormale recipient gender combination as marker for one group of minor histocompatbility antigens, the HY complex and ignored other minor antigens. ${ }^{23,24}$ On the basis of these well-established markers, we postulated a donor type hierarchy and found it confirmed. We had no details on prevention and treatment or on onset and degree of acute and chronic graft-versus-host disease, and did not compare patients with or without graft-versus-host disease. However, 'allogeneic effects' are required for graft-versushost disease, and graft-versus-host disease is the main contributor to non-relapse mortality; allogeneic effects are absent in autologous HSCT. ${ }^{1,25}$ Therefore, we considered the excess of 
Table 3. Estimates of Survival Probabilities at 60 months and Cumulative Excess Event Rates (CEER) of 32,838 patients with allogeneic HSCT by donor type compared to 59,692 patients with autologous HSCT in Europe from 1999 to 2006

\begin{tabular}{|c|c|c|c|c|c|c|}
\hline \multirow[t]{2}{*}{ Donor type $e^{\mathrm{a}}$} & \multicolumn{2}{|c|}{ OS } & \multicolumn{2}{|c|}{ RFS } & \multicolumn{2}{|c|}{$N R M$} \\
\hline & Cox & CEER ${ }^{\mathrm{b}}$ & Cox & $C E E R^{\mathrm{b}}$ & $\operatorname{Cox}^{\mathrm{c}}$ & CEER $R^{\mathrm{b}}$ \\
\hline Syngeneic & 0.69 & -0.10 & 0.55 & -0.20 & n.a. & -0.03 \\
\hline HLA-IdSib, HY' & 0.54 & 0.14 & 0.43 & 0.04 & n.a. & 0.24 \\
\hline HLA-IdSib, $\mathrm{HY}^{+}$ & 0.49 & 0.24 & 0.40 & 0.13 & n.a. & 0.34 \\
\hline Matched Unrel, $\mathrm{HY}^{+}$ & 0.42 & 0.41 & 0.33 & 0.30 & n.a. & 0.53 \\
\hline Mismatched Unrel & 0.37 & 0.51 & 0.30 & 0.42 & n.a. & 0.61 \\
\hline Mismatched Rel & 0.31 & 0.69 & 0.26 & 0.57 & n.a. & 0.78 \\
\hline Spearman correlation & & 0.976 & & 0.976 & & 0.964 \\
\hline$P$-value & & $<0.00$ & & $<0.00$ & & $<0.00$ \\
\hline
\end{tabular}

Abbreviations: NRM, non-relapse mortality; OS, overall survival; RFS, relapse-free survival. All estimates were adjusted for calendar year, conditioning, disease, disease stage, center experience, center size and evaluated at covariate means. Probabilities are presented by a Cox Model stratified by donor type. NRM, nonrelapse mortality (for definitions see Gratwoh ${ }^{20}$ ). ${ }^{\text {a }}$ For definitions see methods section. ${ }^{b}$ Spearman correlation coefficients and tests for significance of systematic increase/decrease with donor type categories. ' n.a. Cox model not applicable, as non-relapse mortality and relapse incidence are competing risks.

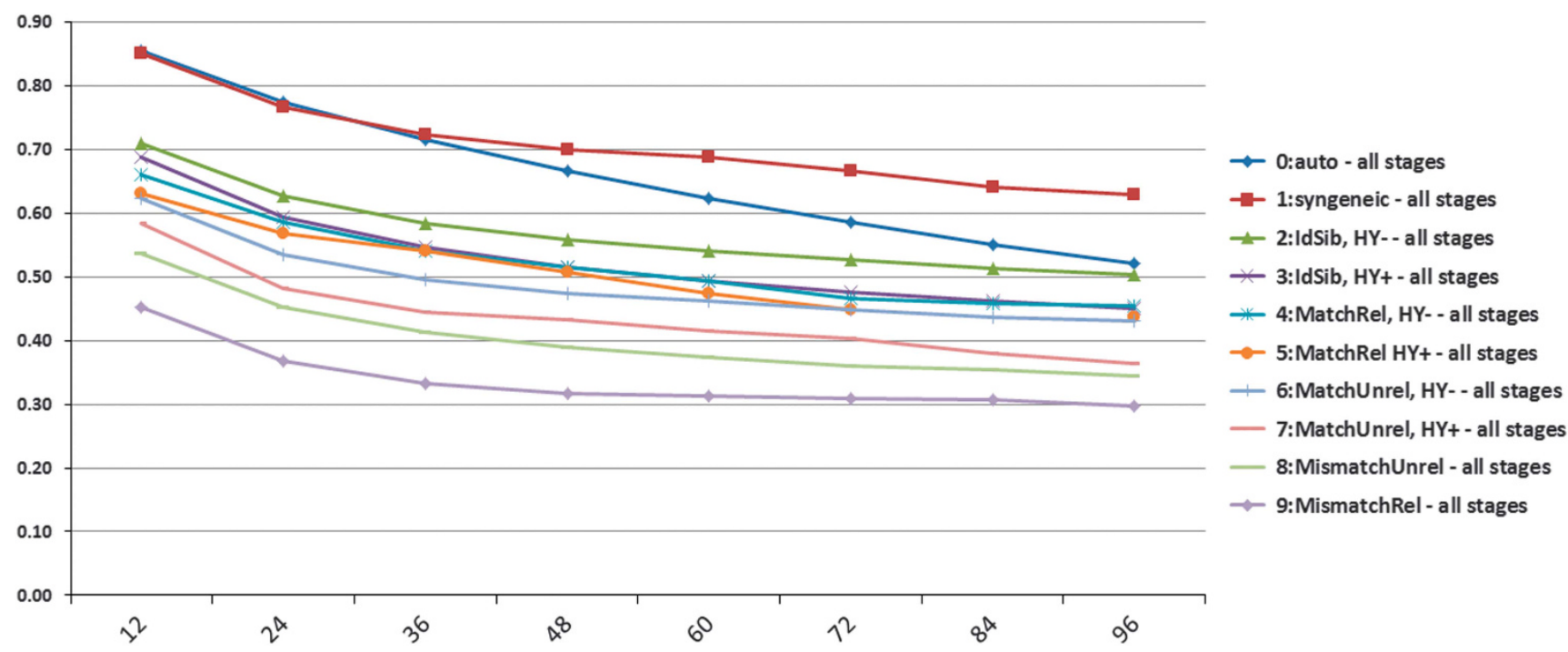

Figure 2. Donor type*and overall survival. The figure depicts outcome of 92,530 patients treated between 1999 and 2006 with an allogeneic $(N=32838 ; 35 \%)$ or autologous $(N=59,692 ; 65 \%)$ hematopoietic stem cell transplant (HSCT) in Europe between 1999 and 2006 . It shows overall survival over 8 years by donor type* using a stratified Cox model for analysis. All estimates adjusted for calendar year, conditioning, disease, stage, center experience, center size and evaluated at covariate means. ${ }^{*}$ for definitions see methods section.

non-relapse mortality, as compared to autologous HSCT as reference group, as an acceptable endpoint and surrogate marker for detrimental alloreactivity. We did not perform a formal analysis of relapse incidence as a marker for graft-versus-malignancy effects. Other effects besides alloreactivity determine relapse, such as genetic disease-related factors, and presence or absence of minimal residual disease. This lack of data, the different types of relapse in the individual disease categories, and the overriding effect of early non-relapse mortality in recipients with increasing HLA-disparity hamper a direct relapse analysis. We did concentrate on non-relapse mortality, and considered the parallel effects on relapse-free and overall survival as estimates for the beneficial effects of alloreactivity.

The patient population was heterogeneous and some subgroups were small. Nevertheless, the analysis did profit from the large predefined cohort. It integrated the key well-described transplant risk factors and adjusted for micro- and macroeconomic risk factors in the analysis. ${ }^{11,19,20}$ The large number of patients, the long follow-up time with a minimum of 8 years, and the systematic pattern in all disease categories and disease stages render it likely that the observations of a donor type hierarchy are true findings.

The results carry major implications. They clarify the role of matching in times when HSCT should provide for each patient a better outcome regarding overall survival, quality of life and costs; ${ }^{19}$ they add caution to current concepts to 'find a donor option for every patient. ${ }^{12-17}$ They fit with recent studies that well-matched unrelated donor HSCT showed results similar to those with matched sibling donors. They suggest complete HLA-typing (12 out of 12 antigens) of unrelated donors ${ }^{26,27}$ before decision-making.

One argument might be that results from our study on transplants performed more than 10 years ago are no longer 
relevant in today's times. Outcome has improved, novel techniques have appeared. ${ }^{17}$ The use of cyclophosphamide shortly after the infusion of stem cell as graft-versus-host disease prophylaxis appears to reduce incidence and severity of graft-versus-host disease. Some early promising results have triggered a rapid expansion in the use of haploidentical donors; still, they await confirmation. ${ }^{12-16}$ Recent results from a large EBMT study with high-risk AML patients indicate a similar outcome at 2 years after haploidentical as compared to 10/10 antigen matched unrelated donor transplants. Non-relapse mortality was reported to be higher, relapse incidence to be lower after haploidentical $\mathrm{HSCT}^{28}$ follow-up was short and risk profiles of the patients different. These early data remain compatible with our findings. The donor type hierarchy prevailed in our long-term analysis regardless of transplant technique. Long-term follow-up is missing with novel techniques. Hence, as of today, if there is a choice, the best match should be donor of choice.

Furthermore, results might redirect research in the present precision medicine initiative on genetic and environmental factors: '..to bring the right prevention and the right treatment for the right patient at the right time' (http://euapm.eu/). Focus has been on factors associated with 'disease' in order to find targets for interventions, ${ }^{29}$ in the context of HSCT with graftversus-host disease. A shift in policies might be indicated towards factors associated with 'no-disease', hence 'no graft-versus-host disease'. About one-third of all HLA-identical sibling donor recipients never ever develop any graft-versus-host disease. ${ }^{30}$ The reasons for absence of graft-versus-host disease are largely unknown. They might be strictly related to differences between donor and recipient in major or minor histocompatibility antigens. There are indications for such constellations. ${ }^{31-33}$ Environmental factors might as well influence presence or absence of graftversus-host disease; the microbiome of the gut has long been in focus and regained most recent interest. ${ }^{33,34}$ If successful, a 'no graft-versus-host disease' constellation might favor an early low cost transplant with no need for immunosuppression post grafting, and save costs and suffering. A high-risk graft-versushost disease constellation might indicate a 'no transplant' treatment strategy.

In summary, our data showed a donor type hierarchy in HSCT where non-relapse mortality decreased stepwise with increasing antigen matching, accompanied by a stepwise increase of overall survival. The beneficial effects of alloreactivity remained, while the detrimental effects decreased. This news might open up new avenues in the era of precision medicine, and launch the search for predictive genomic and environmental factors for 'no graftversus-host disease'.

\section{CONFLICT OF INTEREST}

The authors declare no conflict of interest.

\section{ACKNOWLEDGEMENTS}

We acknowledge the cooperation of the participating teams and their staff: the JACIE Accreditation Office in Barcelona, the JACIE Board and Executive Committee, the JACIE Medical Directors, the JACIE Accreditation Committee, all JACIE inspectors and EBMT registered transplant programs; the EBMT presidents and the working party chairs; the EBMT statistical office in Leiden; the EBMT Co-ordination offices in Barcelona, Paris and London, the Austrian Registry (ASCTR), the Czech BMT Registry, the French Registry (SFGM-TC), the German Registry (DRST), the Italian Registry (GITMO), the Dutch Registry (HOVON), the Spanish BMT Registry (GETH), the Swiss Registry (SBST), the Turkish BMT Registry and the British Registry (BSBMT). JFA acknowledges the support of the NIHR Biomedical Research Centre at Imperial College Healthcare NHS Trust. The study was funded by the European Group for Blood and Marrow Transplantation EBMT, by the Joint Accreditation Committee of EBMT and ISHAGE-Europe JACIE, and the European Leukemia Net ELN. EBMT is supported by grants from the corporate members: Amgen Europe, ViroPharma Europe, Celegene International SARL, Genzyme Europe BV, Gilead Sciences Europe
Ltd, MiltenylBiotec GmbH, Schering-Plough International Inc., Bristol Myers Squibb, CaridiaBCT Europe NV, Cephalon Europe, F Hoffmann-La Roche Ltd, Fresenius Biotech $\mathrm{GmbH}$, TherakosInc., Alexion Europe, ChugaiSanofi-Aventis, Merck Sharp and Dohme, Novartis, Pfizer, Pierre Fabre Médicament.

\section{AUTHOR CONTRIBUTIONS}

AG and RB designed the study concept. PD, RD, HTG, NK, FL, SM, AN and JS were responsible for the integrity of the data. EMG assisted with the collection of the specific microeconomic data. RB performed the statistical analysis. AG, $\mathrm{RB}, \mathrm{AS}, \mathrm{JC}, \mathrm{JA}$ and DN drafted the manuscript. All authors have contributed to the writing, have seen the last version, and have approved of it. Writing of the manuscript was the sole responsibility of the authors.

\section{REFERENCES}

1 Copelan EA. Hematopoietic stem-cell transplantation. N Engl J Med 2006; 354: 1813-1826.

2 Gratwohl A, Baldomero $\mathrm{H}$, Aljurf M, Pasquini MC, Bouzas LF, Yoshimi A et al. Hematopoietic stem cell transplantation: a global perspective. JAMA 2010; 303: 1617-1624.

3 Sureda A, Bader P, Cesaro S, Dreger P, Duarte RF, Dufour C et al. Indications for allo- and auto-SCT for haematological diseases, solid tumours and immune disorders: current practice in Europe. Bone Marrow Transplant 2015; 50: 1037-1056.

4 Majhail NS, Farnia SH, Carpenter PA, Champlin RE, Crawford S, Marks DI et al. Indications for autologous and allogeneic hematopoietic cell transplantation: guidelines from the American Society for Blood and Marrow Transplantation. Biol Blood Marrow Transplant 2015; 21: 1863-1869.

5 Van Bekkum DW. The double barrier in bone marrow transplantation. Semin Hematol 1974; 11: 325-340.

6 Petersdorf EW. The major histocompatibility complex: a model for understanding graft-versus-host disease. Blood 2013; 122: 1863-1872.

7 Magenau J, Runaas L, Reddy P. Advances in understanding the pathogenesis of graft-versus-host disease. Br J Haematol 2016; 173: 190-205.

8 Von Melchner $\mathrm{H}$, Bartlett PF. Mechanisms of early allogeneic marrow graft rejection. Immunol Rev 1983; 71: 31-56.

9 Kongtim P, Cao K, Ciurea SO. Donor specific anti-HLA antibody and risk of graft failure in haploidentical stem cell transplantation. Adv Hematol 2016; 2016: 4025073.

10 Mavroudis D, Barrett J. The graft-versus-leukemia effect. Curr Opin Hematol 1996; 3: 423-429.

11 Bortin MM. A compendium of reported human bone marrow transplants. Transplantation 1970; 9: 571-587.

12 Passweg JR, Baldomero H, Bader P, Bonini C, Cesaro S, Dreger P et al. Hematopoietic SCT in Europe 2013: recent trends in the use of alternative donors showing more haploidentical donors but fewer cord blood transplants. Bone Marrow Transplant 2015; 50: 476-482.

13 Worel N, Buser A, Greinix HT, Hägglund H, Navarro W, Pulsipher MA et al. Suitability criteria for adult related donors: A Consensus Statement from the Worldwide Network for blood and marrow transplantation standing committee on donor issues. Biol Blood Marrow Transplant 2015; 21: 2052-2060.

14 Ciurea SO, Zhang MJ, Bacigalupo AA, Bashey A, Appelbaum FR, Aljitawi OS et al. Haploidentical transplant with posttransplant cyclophosphamide vs matched unrelated donor transplant for acute myeloid leukemia. Blood 126: 1033-1040.

15 Anasetti C. Use of alternative donors for allogeneic stem cell transplantation. Hematology. Am Soc Hematol Educ Program 2015; 2015: 220-224.

16 Milano F, Gooley T, Wood B, Woolfrey A, Flowers ME, Doney K et al. Cord-blood transplantation in patients with minimal residual disease. New Engl J Med 2016; 375: 944-953.

17 Apperley J, Niederwieser D, Huang XJ, Nagler A, Fuchs E, Szer J et al. Haploidentical hematopoietic stem cell transplantation: a global overview comparing Asia, the European Union, and the United States. Biol Blood Marrow Transplant 2016; 22: 23-26.

$18 \mathrm{https}: / / w w w . w h i t e h o u s e . g o v /$ the-press-office/2015/01/30/fact-sheet-presidentobama-s-precision-medicine-initiative. Accessed on 10 February 2017.

19 Gratwohl A, Sureda A, Baldomero H, Gratwohl M, Dreger P, Kröger N et al. Economics and outcome after hematopoietic stem cell transplantation: a retrospective cohort study. EBioMedicine 2015; 2: 2101-2109.

20 Gratwohl A. The EBMT risk score. Bone Marrow Transplant 2012; 47: 749-756.

21 Sorror ML, Sandmaier BM, Storer BE, Maris MB, Baron F, Maloney DG et al. Comorbidity and disease status based risk stratification of outcomes among 
patients with acute myeloid leukemia or myelodysplasia receiving allogeneic hematopoietic cell transplantation. J Clin Oncol 2007; 25: 4246-4254.

22 Singer RB. Comparative Morbidity- What are the Prospects? In Medical risks. Compend of Morbidity and Mortality. Singer RB, Kita MW, Avery JR (eds). The American Academy of Insurance Medicine: Ottawa, ON, Canada, 1991; pp 84-88. Available at: http://www.aaimedicine.org/contact-us/default.asp.

23 Spierings E. Minor histocompatibility antigens: past, present, and future. Tissue Antigens 2014; 84: 374-60

24 Nakasone H, Tian L, Sahaf B, Kawase T, Schoenrock K, Perloff S et al. Allogeneic HY antibodies detected 3 months after female-to-male HCT predict chronic GVHD and nonrelapse mortality in humans. Blood 2015; 125: 3193-3201.

25 Kaffenberger J, Basak SA, loffreda M. Autologous graft-versus-host disease in a child with stage IV neuroblastoma. Pediatr Dermatol 2015; 32: 276-279.

26 Lee SJ, Klein J, Haagenson M, Baxter-Lowe LA, Confer DL, Eapen M et al. Highresolution donor-recipient HLA matching contributes to the success of unrelated donor marrow transplantation. Blood 2007; 110: 4576-4583.

27 Cornelissen JJ, Blaise D. Hematopoietic stem cell transplantation for patients with AML in first complete remission. Blood 2016; 127: 62-70.

28 Versluis J, Labopin M, Ruggeri A, Socié G, Wu D, Volin L et al. 2017. Alternative donors for hematopoietic stem cell transplantation in poor risk AML in CR1. Blood Advan 2017, 1: 477-485.

29 Lek M, Karczewski KJ, Minikel EV, Samocha KE, Banks E, Fennell T et al. Analysis of protein-coding genetic variations in 60,706 humans. Nature 2016; 536: 285-291.

30 Gratwohl A, Ruiz de Elvira C, Gratwohl M, Greinix HT, Duarte R. Gender and graftversus-host disease after hematopoietic stem cell transplantation. Biol Blood Marrow Transplant 2016; 22: 1145-1146.
31 Morishima S, Kashiwase K, Matsuo K, Azuma F, Yabe T, Sato-Otsubo A et al. High-risk HLA alleles for severe acute graft-versus-host disease and mortality in unrelated donor bone marrow transplantation. Haematologica 2016; 101: 491-498.

32 Dickinson AM, Norden J. Non-HLA genomics: does it have a role in predicting haematopoietic stem cell transplantation outcome? Int J Immunogenet 2015; 42: 229-238.

33 Petersdorf EW, Malkki M, O'hUigin C, Carrington M, Gooley T, Haagenson MD et al. High HLA-DP expression and graft-versus-host disease. N Engl J Med 2015; 373: 599-609.

34 Mathewson ND, Jenq R, Mathew AV, Koenigsknecht M, Hanash A, Toubai T et al. Gut microbiome-derived metabolites modulate intestinal epithelial cell damage and mitigate graft-versus-host disease. Nat Immunol 2016; 17: 505-513.

(i) $⿴$ This work is licensed under a Creative Commons Attributioncc) NonCommercial-NoDerivs 4.0 International License. The images or other third party material in this article are included in the article's Creative Commons license, unless indicated otherwise in the credit line; if the material is not included under the Creative Commons license, users will need to obtain permission from the license holder to reproduce the material. To view a copy of this license, visit http:// creativecommons.org/licenses/by-nc-nd/4.0/

(C) The Author(s) 2017

Supplementary Information accompanies this paper on the Leukemia website (http://www.nature.com/leu) 